\title{
A Novel Fast and Efficient Approach to Purify the Thrombin-like Enzyme from Two Bothrops-genus Snake Venoms
}

\author{
Mauricio Aurelio Gomes Heleno ${ }^{1,2, *}$, Edda Evnet Newball-Noriega ${ }^{3}$, Salomón Huancahuire-Vega ${ }^{3}$, \\ Rui Seabra Ferreira Júnior ${ }^{1,2}$, Benedito Barraviera ${ }^{1,2}$ \\ ${ }^{1}$ Center for the Study of Venoms and Venomous Animals (CEVAP), Univ Estadual Paulista (UNESP), Botucatu/SP, Brazil \\ ${ }^{2}$ Botucatu Medical School, Univ Estadual Paulista (UNESP), Botucatu/SP, Brazil \\ ${ }^{3}$ Faculty of Health Sciences, School of Human Medicine, Universidad Peruana Unión (UPeU), Lima, Perú
}

Email address:

heleno.cevap@yahoo.com.br (M. A. G. Heleno)

${ }^{*}$ Corresponding author

\section{To cite this article:}

Mauricio Aurelio Gomes Heleno, Edda Evnet Newball-Noriega, Salomón Huancahuire-Vega, Rui Seabra Ferreira Júnior, Benedito Barraviera. A Novel Fast and Efficient Approach to Purify the Thrombin-like Enzyme from Two Bothrops-genus Snake Venoms. American Journal of Biomedical and Life Sciences. Vol. 9, No. 4, 2021, pp. 209-218. doi: 10.11648/j.ajbls.20210904.15

Received: June 24, 2021; Accepted: July 20, 2021; Published: August 31, 2021

\begin{abstract}
Snake venoms are important sources of complex substances with a variety of pharmacological activities. Among them serine proteinases (SVSPs) have important effects on the hemostatic system influencing the hemodynamic of human or animal blood. Bothrops genus-snake venoms are rich in the thrombin-like enzyme, a type of SVSPs, with great interest to produce medicine. Therefore, the aim of this work was to describe a rapid, only two-step chromatographic-procedure developed to perform a faster purification of SVSPs from Bothrops alternatus and Bothrops moojeni venoms. As a result, two groups of serine proteinases respectively BaIII-4 -8 and BmIII-2 - 5, were isolated and their molecular masses estimated by mass spectrometry and SDS-PAGE under denaturing conditions. The SVTLEs isolated from B. alternatus $($ BaIII-3 - 8) and $B$. moojeni (BmIII-2 - 5) fractions displayed apparent molecular mass around 30-40 $\mathrm{kDa}$ which closely relates to SVTLEs from other Bothrops species, as well their amino acid partial sequence triptych ions. Analysis of the alignment of the amino acid residue sequences of the N-terminal of the isolated proteins revealed a high level of identity with other SVTLEs. These enzymes coagulated plasma and showed fibrinogenolytic activity in blood. These SVTLEs isolated can be considered $\alpha-$ fibrinogenase mainly due to the fact that they hydrolyze the A $\alpha$ chain fibrinogen. $B$. moojeni SVTLE showed greater activity than those from $B$. alternatus isolated. This new purification alternative approach developed was faster and more economical than the traditional process currently used. Faster purification and improved extraction yield can provide new insights into these enzymes including the use as a candidate molecule in the production of new drugs.
\end{abstract}

Keywords: Bothrops alternatus, Bothrops moojeni, Serine Proteinases, Thrombin-like Enzyme, Fast Purification

\section{Introduction}

Bothrops snake venoms contain a large variety of proteins and peptides affecting the hemostatic system; these proteins are classified as coagulant, anticoagulant or fibrinolytic factors [4, 44, 46]. Among these, a group of serine proteinases enzymes convert fibrinogen into fibrin by cleaving fibrinopeptides A and/or B. As this activity resembles the activity of thrombin, these venom components are commonly named "thrombin-like" enzymes (SVTLEs) $[41,43]$. These proteins are good tools to study molecular details of activation of specific factors involved in coagulation and fibrinolytic cascades. They are also useful in treating various thrombotic and hemostatic conditions after a medicine developed [9, 28]. In addition to affecting the hemostatic system these proteins affect platelet aggregation, 
complement system, blood pressure and nervous system [23, 34].

The SVTLEs share many biochemical and structural properties such as a conserved catalytic triad (Ser195, His57, Asp102), and their three-dimensional structure is highly conserved. Their structure, taken as a whole, is made of two $\beta$-barrels constituted of six-strands and separated by the catalytic residues, and of a C-terminal helical segment [41]. Moreover, they are glycoproteins with a single chain and molecular mass between 28 to $60 \mathrm{kDa}$ and they are active on thrombin-specific synthetic substrates. SVTLEs have a high degree of homology among them, approximately 60 to $68 \%$, but show less than $40 \%$ homology with human endogenous thrombin [38].

Bothrops snakes have their venom richly comnposed of SVTLE, several of which have already been purified and characterized: B. atrox [45], B. alternatus [11], B. pauloensis [10], B. moojeni [4], B. jararacussu [19], B. jararaca [42], B. brazili [46] and recently $B$. pictus [43]. The isolation of SVTLEs is of great interest to the scientific community due to the possibility of their use in the treatment of thrombotic diseases and as anticoagulants, in addition to their relevance as a target for the bioprospecting of new products [25].

Within this context, in the early 1990s, the Center for the Study of Venoms and Venomous Animals (CEVAP) at São Paulo State University (UNESP), Brazil initiated an innovative research for the production of a new fibrin sealant from snake venom serine proteinases. The sealant was made from the replacement of bovine/human thrombin by thrombin-like gyroxin, purified from the venom of Crotalus durissus terrificus (Southamerican Rattlesnake) [6, 8, 13, 18, 20, 33]. Curiously, a systematic review about heterologous biopolymers with hemostatic, adhesive, sealant, scaffold, and drug delivery properties, the one produced by CEVAP/UNESP has been the only one found so far $[1,7,12$, 14]. In addition to gyroxin, other thrombin-like enzymes especially from Bothrops-genus could be employed in the composition of this unique biopolymer after being purifying and standardizing the biological activities.

The SVTLEs purification processes that have been reported so far involve three or more chromatographic steps and, in several cases, their performance and recovery are considered low $[17,24,26]$. Consequently, to obtain a purified protein pool using several purification steps leads to a loss of approximately $30 \%$ of the material in each step performed. Therefore, it is important that an efficient method is deployed, standardized, and validated for the chromatographic purification of other thrombin-like enzymes such as those found in B. alternatus and B. moojeni snake venoms.

Aiming at a higher yield this work aimed to search a new fast and optimized process combining only two chromatographic techniques that allow the isolation and consequently the characterization of these thrombin-like enzymes. In the end, structural analysis and biological activities were compared with other SVTLEs that have already been deposited in databases.

\section{Methods}

\subsection{Venom}

The venoms were obtained from a venom-pool had been milked from Bothrops snakes (B. alternatus and B. moojeni) adults, of both sexes, individually microchipped, created and maintained in the CEVAP's Serpentarium, located at CEVAP-UNESP, Botucatu, São Paulo Brazil, according to the methodology developed by this Translational Center (https://youtu.be/CPcs4ity-Uw).

\subsection{Purification Method}

Five hundred miligrans of whole venom were obtained from each pool of venoms of two Bothrops genus studied were applied to a Benzamidine-Seharose $6 \mathrm{~B}$ affinity column in a ÄKTA Explorer 100 liquid chromatography system $\mathrm{pH}$ 7.4 (GE Healthcare, Diegem, Belgium), previously equilibrated with $0.05 \mathrm{M}$ Tris-HCl buffer, $\mathrm{pH} 7.4$ (buffer 1). Elution was performed with $30 \mathrm{~mL}$ of $0.05 \mathrm{M}$ Tris- $\mathrm{HCl} \mathrm{pH}$ $7.4+0.5 \mathrm{M} \mathrm{NaCl}$ (buffer 2) and $20 \mathrm{~mL}$ of $0.02 \mathrm{M}$ glycine $\mathrm{pH}$ 3.2 (buffer 3) with fractions eluted in glycine were neutralized directly in the collection tube with $400 \mu \mathrm{L}$ of 1.0 $\mathrm{M}$ Tris- $\mathrm{HCl} \mathrm{pH}$ 9.0. The collection flow was $30 \mathrm{~mL} /$ hour, with $3.0 \mathrm{~mL} /$ tube collected at a temperature of $25^{\circ} \mathrm{C}$.

The fractions isolated by affinity chromatography were diluted in $250 \mu \mathrm{L}$ of $0.1 \%(\mathrm{v} / \mathrm{v})$ trifluoroacetic acid (TFA) and subjected to a High Performance Liquid Chromatography system (Shimadzu, Kyoto, Japan) on a reverse phase column (RP-HPLC) C18 (20 x $250 \mathrm{~mm})$. The sample was initially eluted in $0.1 \%(\mathrm{v} / \mathrm{v})$ trifluoroacetic acid (TCA), followed by a linear gradient of $70 \%$ acetonitrile in a flow of $1 \mathrm{~mL} / \mathrm{min}$ at room temperature. The peak of each serine proteinase in question was separated and lyophilized for further characterization.

\subsection{In Vitro Coagulant Activity Assay}

The process of purifying TLE serine proteinases was monitored by coagulant activity on blood. Blood samples were collected from healthy donors in the presence of $3.8 \%$ sodium citrate, in the proportion of 9:1 and centrifuged at $2500 \mathrm{xg}$ at $4^{\circ} \mathrm{C}$ for 15 minutes to obtain plasma. Coagulant activity was performed using $200 \mu \mathrm{L}$ of citrated human plasma incubated with $25 \mu \mathrm{g}$ of each of the serine proteinases isolated from the snake venoms, diluted in milliQ water at a concentration of $1 \mathrm{mg} / \mathrm{mL}$. This mixture was incubated in a water bath at $37^{\circ} \mathrm{C}$ and the time of formation of the fibrin network was timed. Each sample assay was performed in triplicate. The maximum observation period for the formation of the fibrin network was five minutes, and after that it was considered as no coagulant activity (Theakston \& Reid, 1983).

\subsection{SDS-PAGE}

SDS-PAGE was performed for homogeneity and determination of relative molecular mass $(\mathrm{Mr})$ evaluation. Electrophoresis was carried out using $13 \%$ polyacrylamide 
gel in Tris- $\mathrm{HCl}$ buffer, pH 8.8 containing $10 \%$ SDS in a Vertical Electrophoresis System (Bio-Rad Laboratories, Inc., Hercules, CA, USA) under denaturing conditions using as molecular weight standards, Phosphorylase B (97 kDa), Bovine Serum Albumin (66 kDa), Ovalbumin (45 kDa) carbonic anhydrase $(30 \mathrm{kDa})$, trypsin inhibitors $(20.1 \mathrm{kDa})$ and $\alpha$-lactalbumin $(14.2 \mathrm{kDa})$. After electrophoretic running, gels were stained with Coomassie Brilliant Blue R-250.

\subsection{Mass Spectrometry}

After running SDS-PAGE gels, the protein bands were excised and subjected to an in-gel trypsin digestion followed by ESI-QUAD-TOF Mass Spectrometry and Mascot (Matrix Science, CO, UK) search engine against the NCBI NR database restricted to the taxa Snakes. For the mass spectrometry analysis, it was used the quadrupole electrospray (ESI) mass spectrometry equipment, model micrOTOF-Q III (Bruker Daltonics, Bremen, Germany), coupled to an LC-20AT liquid chromatograph (Shimadzu, Kyoto, Japan). The liquid chromatography was coupled to the mass spectrometry equipment, composed of a binary pump system and an automatic sample applicator. The mobile phase consisted of water (A) and acetonitrile (B), containing $0.1 \%(\mathrm{v} / \mathrm{v})$ formic acid or $0.1 \%(\mathrm{v} / \mathrm{v})$ TFA. In addition, chromatographic separation was performed using a $\mathrm{C} 18$ reverse phase column $(4.5 \mathrm{~mm} \times 100 \mathrm{~mm}, 1.8 \mu \mathrm{m})$. The elution conditions were optimized in a linear gradient from 0 to $85 \%$ of solvent $\mathrm{B}$ for 60 minutes, in a flow of $0.2 \mathrm{~mL} / \mathrm{min}$ The column and the automatic sample applicator were maintained at $25^{\circ} \mathrm{C}$ and $10^{\circ} \mathrm{C}$, respectively. The injection volume of the reference compounds and samples obtained by digestion in-gel was $2 \mu \mathrm{L}$.

MASCOT v.2.1 server (www.matrixscience.com) was used as a bioinformatic tool to assist identification of the isolated protein samples. The protein and fragmentation spectra obtained experimentally by mass spectrometry were compared with the set of peptide sequences allocated in the SwissProt database. To increase the precision of the identification processsof proteins, the following parameters were used: Enzyme: Trypsin as a cleavage agent; Taxonomy: Snakes; Carbamidomethylation (cysteine) as a fixed modification; Oxidation (methionine and tryptophan) as a variable modification; Number of cleavages lost by the enzyme: 1; Molecular weight of type: Monoisotopic; Molecular weight: without restrictions; Peptide tolerance error $\pm 0.5 \mathrm{Da}$ and MS / MS tolerance error $\pm 0.8 \mathrm{Da}$ for MS / MS analysis; Protonation: $+1,+2,+3$ for the state of the peptide charge; Instrument type: ESI-Q-ToF.

\subsection{N-terminal Amino Acids Sequence}

The analysis of the $\mathrm{N}$-terminal sequence of each purified serine proteinase was performed in an automatic protein sequencer Shimadzu model PPSQ-23A (Shimadzu, Kyoto,
Japan). A solution with approximately $1 \mathrm{mg} / \mathrm{mL}$ of the enzyme was applied to the sequencer and the amino acids sequence was determined by the Edman degradation method [16]. After determining the sequence of each serine proteinase, their amino acid homology was compared with other serine proteinases, by alignment using the BLAST protein program [2]. N-terminal sequence alignments of purified serine proteinases with others thrombin-like serine proteinases were made using ClustalW in Edit Seq 5.01 (C) DNASTAR (Madison, WI., USA).

\subsection{Fibrinogenolytic Activity}

Fibrinogenolytic activity was determined according to the methodology described by Rodrigues et al [30]. Samples containing $30 \mu \mathrm{g}$ of bovine fibrinogen were incubated with $10 \mu \mathrm{g}$ of the purified proteins at $37^{\circ} \mathrm{C}$ for $2 \mathrm{~h}$. The reaction was stopped by adding $10 \mu \mathrm{L}$ of sample buffer $0.05 \mathrm{M}$ Tris$\mathrm{HCl}$, pH 8.0 containing glycerol $10 \%$ (v / v), $\beta$ mercaptoethanol $10 \%(\mathrm{v} / \mathrm{v})$, SDS $2 \%(\mathrm{v} / \mathrm{v})$ and $0.05 \%$ bromophenol blue $(\mathrm{w} / \mathrm{v})$, followed by boiling at $100^{\circ} \mathrm{C}$ for 5 minutes. Then the samples were analyzed by $13 \%$ SDSPAGE.

\section{Results}

\subsection{Purification of Thrombin Like Enzymes from $B$. Alternatus and B. moojeni Venoms}

The elution profiles of $B$. alternatus and B. moojeni venoms subjected to an affinity chromatography (benzamidine-sepharose 6B column) showed 3 fractions each (BaI-III andBmI-III, respectively) (Figure 1A, B). A coagulant activity was observed in the fraction III of both venoms. After asecond chromatographic step was performed (reverse phase HPLC system, using an analytical column C18), the fractionation of BaIII resulted in 8 peaks (BaIII-1 to 8). Peaks BaIII-4 to BaIII-8 showed coagulant activity (Figure 1B). On the other hand, the BmIII fraction revealed 5 peaks (BmIII-1 to 5) and a coagulant activity was observed for peaks BmIII-2 to BmIII-5 (Figure 1D). These peaks were then selected for the analysis of mass spectrometry, determination of the N-terminal sequence and fibrinogenolytic activity.

The inserts in figures 1B-D show the presence of a single electrophoretic band of all proteins purified under denaturing conditions, with relative molecular mass around $35 \mathrm{kDa}$ in relation to the molecular mass markers (lane 1). These results indicate a high degree of homogeneity of each fraction. Table 1 shows a summary of the steps involved in the purification process and protein recovery, with BaIII-6, BmII-2 and BmIII-4 having the best performance $(36.4 \%, 31.2 \%$ and $51.5 \%$ respectively). 

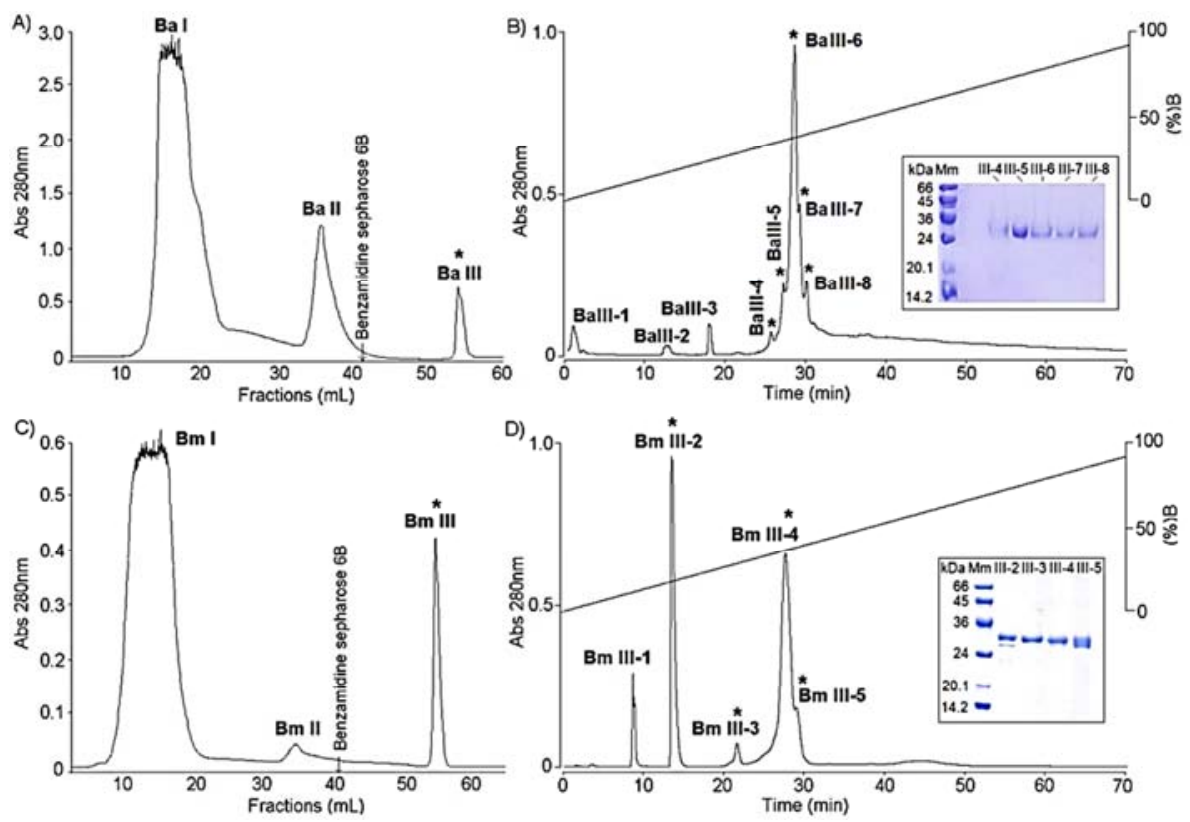

Figure 1. Elution profile of B. alternatus $(A)$ and B. moojeni $(C)$ venoms by Benzamidine-Seharose $6 B$ affinity column. In each venom the coagulant activity was located in peak III (Ba III and Bm III, respectively). Subsequently, the fractions III of each venom, Ba III from B. alternatus (B) and Bm III from B. moojeni (D) were subjected to RP-HPLC in a Shimadzu C18 column. *Fractions BaIII-4, BaIII-5, BaIII-6, BaIII-7, Ba III8 from B. alternatus and BmIII-2, BmIII-3, BmIII-4 and BmIII-5 from B. moojeni contained coagulant activity. Inserts: Electrophoretic profiles on SDS-PAGE (13\%) under denaturing and reducing conditions (Mm: molecular mass markers).

Table 1. Parameters of B. alternatus and B. moojeni thrombin-like serine proteinases purification procedure.

\begin{tabular}{|c|c|c|c|c|c|c|}
\hline Venom & $\begin{array}{l}\text { Total venom mass used (mg) } \\
\text { to affinity chromatography }\end{array}$ & $\begin{array}{l}\text { Protein dosage (mg) } \\
\text { after affinity } \\
\text { chromatography }\end{array}$ & $\begin{array}{l}\text { Yield (\%) after } \\
\text { affinity } \\
\text { chromatography }\end{array}$ & $\begin{array}{l}\text { Protein mass (mg) } \\
\text { after RP-HPLC }\end{array}$ & $\begin{array}{l}\text { Yield (\%) after } \\
\text { RP-HPLC }\end{array}$ & $\begin{array}{l}\text { Fractions } \\
\text { purification (n } \\
\text { times) }\end{array}$ \\
\hline \multirow{5}{*}{$\begin{array}{l}\text { Bothrops } \\
\text { alternatus }\end{array}$} & \multirow{5}{*}{500} & \multirow{5}{*}{14,6} & \multirow{5}{*}{2,92} & BaIII-4=1,04 & 7,1 & 480 \\
\hline & & & & BaIII-5=5,32 & 36,4 & 94 \\
\hline & & & & BaIII- $6=0,82$ & 5,6 & 609 \\
\hline & & & & BaIII-7=1,01 & 6,8 & 495 \\
\hline & & & & BaIII- $8=0,32$ & 2,2 & 1562 \\
\hline \multirow{3}{*}{$\begin{array}{l}\text { Bothrops } \\
\text { moojeni }\end{array}$} & \multirow{3}{*}{500} & \multirow{3}{*}{32,0} & \multirow{3}{*}{6,4} & BmIII-3=1,98 & 6,2 & 252 \\
\hline & & & & BmIII-4=16,5 & 51,5 & 30 \\
\hline & & & & BmIII-5=2,76 & 8,6 & 181 \\
\hline
\end{tabular}

\subsection{Identification of Peptides by Mass Spectrometry of Thrombin Like Enzymes from $B$. alternatus and $B$. moojeni Venoms}

Proteins bands were excised from SDS-PAGE gels and ingel trypsin digestion was performed followed by ESIQUAD-TOF Mass Spectrometry and Mascot search engine against the NCBI NR database restricted to the taxa Snakes.
Table 2 shows serine proteinases identified from the peptide fragments analyzed by mass spectrometry.

Crotalus adamanteus, Trimeresurus albolabris, Agkistrodon bilineatus, B. jararaca and B. atrox were the species of snakes to which the obtained serine proteinases were compared. The identified peptides have an ion score greater than 34 , which indicates identity or a high degree of similarity.

Table 2. Data of peptides obtained from SDS-PAGE gels protein bands submitted to trypsin digestion and identified by ESI-QUAD-TOF Mass Spectrometry.

\begin{tabular}{|c|c|c|c|c|c|c|c|c|}
\hline Sample & $\begin{array}{l}\text { Protein name } \\
\text { (snake databank) }\end{array}$ & Access Code & Taxonomy & $\begin{array}{l}\text { Mascot/Ion } \\
\text { Scores } \\
\end{array}$ & $\begin{array}{l}\text { Observed } \\
\text { Ion }\end{array}$ & Mass & $\begin{array}{l}\text { Charge } \\
\mathbf{3}(+)\end{array}$ & Peptides (Ion Score) \\
\hline BaIII-4 & SVSP3 & gi|338855336 & C. adamanteus & $50 / 43$ & 560.7975 & 29719 & 4 & R.AAKPELPATSRTLCAGILEGGK.G \\
\hline BaIII-5 & GPV-PA & gi|380875424 & T. albolabris & $39 / 41$ & 553.276 & 29094 & 2 & K.TLNEDEQTR.D \\
\hline BaIII-6 & GPVPA & gi|380875424 & T. albolabris & $44 / 45$ & 553.271 & 29094 & 2 & K.TLNEDEQTR.D \\
\hline BaIII-7 & SVSP & gi|461511 & A. bilineatus & $27 / 27$ & 500.2358 & 2247 & 3 & VVGGDECNINEHR.S \\
\hline BaIII-8 & GPV-PA & gi|380875424 & T. albolabris & $49 / 38$ & 553.2734 & 29094 & 2 & K.TLNEDEQTR.D \\
\hline BmIII-2 & PABJ & gi|999161 & B. jararaca & $114 / 49$ & 404.2194 & 25883 & 3 & K.INILDHAVCR.A \\
\hline BmIII-4 & Batroxobin & gi|62464 & B. atrox & $106 / 47$ & 477.6094 & 28854 & 3 & K.NVITDKDIMLIR.L \\
\hline BmIII-5 & Batroxobin & gi|62464 & B. atrox & $142 / 53$ & 477.6055 & 28854 & 3 & K.NVITDKDIMLIR.L \\
\hline
\end{tabular}




\subsection{Determination of $\mathrm{N}$-terminal Amino Acid Sequence of Thrombin Like Enzymes from B. alternatus and $B$. moojeni Venoms}

Table 3. Sequencing of the amino acids N-terminal portion of TLEs purified from B. alternatus and B. moojeni venoms.

\begin{tabular}{ll}
\hline Sample & Sequencing of the amino acids N-terminal \\
\hline B. alternatus & \\
BaIII-4 & QIGGEEWNINEHRSLVVLFQEQRQL \\
BaIII-5 & QIGGDEENIIEERRLVVIFFSSGFF \\
BaIII-6 & QIGGEEEEINNEERLLVVVVFFTGG \\
BaIII-7 & QIGGEEENINEERSLVAAAFSTGFF \\
BaIII-8 & QIGGDEENNNNHRSLVAAFFSSSFF \\
B. moojeni & \\
BmIII-2 & VIGGRPIKINKHRRLVVLFKSSSLL \\
BmIII-3 & IGGRPIKINKHRSLVVLFTSSSLL \\
BmIII-4 & VLGGDPADLNYHPFFAAMMYSP \\
BmIII-5 & VIGGREEKIIKHHSLLLLLYYYGLL \\
\hline
\end{tabular}

Analysis of N-terminal amino acid sequence of the serine proteinases obtained from $B$. alternatus and B. moojeni was carried out using the Edman degradation method. The results

showed that between 22 to 25 amino acids from the $\mathrm{N}$ terminal region were sequenced, as shown in table 3.

\subsection{Alignment of the $N$-terminal Sequences of the Thrombin Like Enzymes Isolated from B. alternatus and B. moojeni Venoms}

Figure 2 shows the alignment of the N-terminal sequences of the serine proteinases isolated from the $B$. alternatus venom, BaIII-4 - 8 with other TLEs from snake venoms deposited in the NCBI Database (Figure 2A - E, respectively), as well as the sequence comparison of these 5 isolated serine proteinases (Figure 2F). The figure 2 also shows the conserved amino acid residues compared to each isolated protein. In the same way, the N-terminal sequence of serine proteinases isolated from $B$. moojeni venom were aligned with sequences of other toxins from the same snake family (Figure 3A - E). BmIII-2 showed a great identity matching with PA-BJ of B. jararaca, BmIII-3 with the serine proteinase 3 of Protobothrops jerdonii, BmIII-4 with Leucurobin of B. leucurus and BmIII-5 with Cerastotin of C. cerastes.

B)

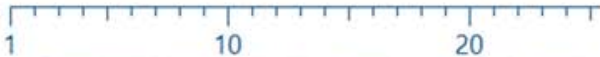

Balli-5 Q I GGDEEN I I EERRLVVIFFSSGFF Asperase - I GGDECN INEHRSLVVLFNSSGF Bhalternin - I GGDECN INEHRSL VVLFNSSGFBpirSP27 - VGGDECNINEHRSLVA I FNSTGFF PATS - VGGDECNINEHRSLVAIFNSTGFF BjSP - VGGDECNINEHRSLVA I FNSTGFF

C)

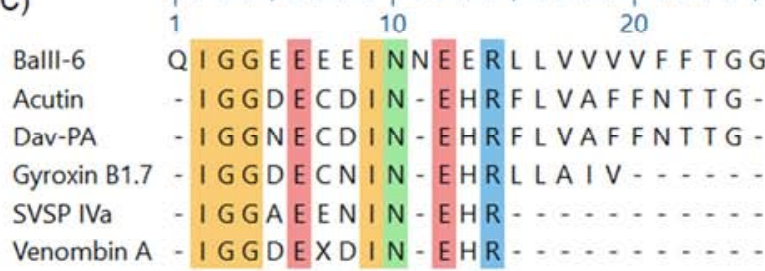

\section{E)}

Balli-8 QIGGDEENNNNHRSLVAAFFSSFF

KR-E1 - I GGDECN INEHRSLVAFFNSTGFF

Saxthrombin - I GGDECN I NEHRS L VAFFNSTGFF

SVSP 8 - I GGDECN INEHRSLVA I FNSTKFF

BpirSP27 - VGGDECN INEHRSLVA I FNSTGFF

PATS $\quad-V G G D E C N$ INEHRSLVAIFNSTGFF
D)

\begin{tabular}{|c|c|}
\hline & 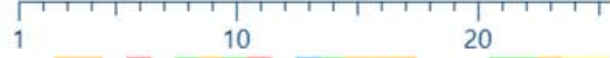 \\
\hline $111-7$ & Q I GGEEENINEERSLVAAAFSTGFF \\
\hline R-E1 & - IGGDECNINEHRSLVAFFNSTGFF \\
\hline xthrombin & - IGGDECN INEHRSLVAFFNSTGFF \\
\hline irSP27 & - VGGDECNINEHRSLVAIFNSTGFF \\
\hline SP 1 & - VGGDECN INEHRSLVA IFNSTGFF \\
\hline HS & - VGGDECN INEHRSLVAIFNSTGFF \\
\hline
\end{tabular}

F)

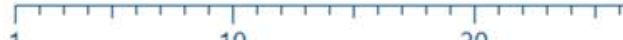

Consensus $Q$ I GGEEEN IN-EERS LVV a F F S X GFF

Balll-4 Q I GGEEWN IN-EHRSLVVLFQEQRQL

Balll-5 Q I GGDEEN I I-EERRLVVIFFSSGFF

Balll-6 QIGGEEEE INNEERLLVVVVFFTGG -

Ball-7 Q I GGEEEN IN-EERSLVAAAFSTGFF

Balll-8 QIGGDEENNN-NHRSLVAAFFSSSFF

Figure 2. Multiple alignments of $N$-terminal amino acids sequences of thrombin-like serine proteinases (TLSP) from B. alternatus venom obtained by the Edman degradation method. (A) BaIII-4, (B) BaIII-5, (C) BaIII-6, (D) BaIII-7, (E) BaIII-8 and (F) BaIII4-8. The N-terminal sequences of other SVSP were obtained from the BLAST-p database and aligned using ClustalW in Edit Seq 5.01 C DNASTAR. (Madison, WI., USA). Colors show only conserved residues compared to reference. LmrSP-4 from L. muta rhombeata (COHLA3.1), SVSP Ca from Crotalus atrox (Q9PRW4.1), SVSP CC from Cerastes cerastes (Q9PRM8), KR-E1 from Gloydius ussuriensis (Q7SZE2.1), TL-BJ2 from B. jararaca (P81883.1), Bilineobin from Agkistrodon bilineatus accesion (Q9PSN3.1), Asperase from B. asper (Q072L6.1), Bhalternin from B. alternatus (P0CG03.1), BpirSP27 from B. pirajai (P0DL26.1), PA Ts from Trimeresurus stejnegeri (Q71QH7.1), BjSP from B. jararaca (Q5W959.1), Acutin from Deinagkistrodon acutus (Q9YGS1.1), Dav-PA from D. acutus (Q9I8X1.2), Gyroxin B1.7 from C. durissus terrificus (BOFXM3.1), SVSP IVa from C. cerastes (Q7LZF4.1), Saxthrombin from Gloydius saxatilis (Q7SZE1.1), SVSP 1 from T. gramineus (O13059.1), SVSP HS114 from B. jararaca (Q5W959.1) and SVSP 8 from C. adamanteus (J3S835.1). 
A)

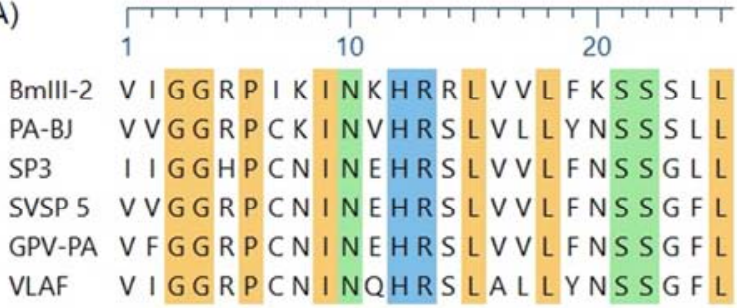

C) $\begin{array}{ll}\text { Bmlll-4 } & \text { VLGGDPADLNYHPFFAAMMYSP } \\ \text { Leucurobin } & \text { VIGGDECDINEHPFLAFMYYSP } \\ \text { Bothrombin } & \text { VIGGDECDINEHPFLAFMYYSP } \\ \text { Batroxobin } & \text { VIGGDECDINEHPFLAFMYYSP } \\ \text { BjussuSP-1 } & \text { VLGGDECDINEHPFLAFLYS - } \\ \text { Barnettobin } & \text { VIGGDECDINEHPFLAFLYS - }\end{array}$
B)

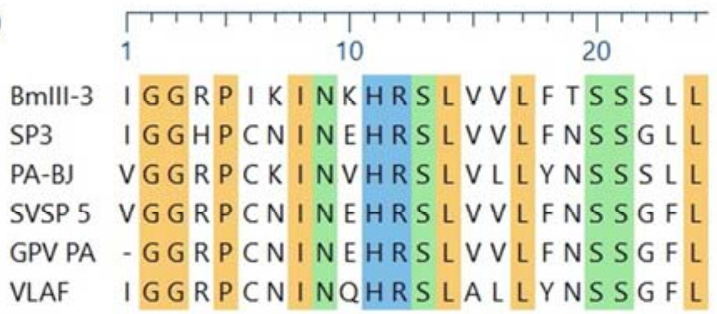

D)

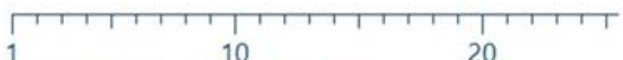

BmIII-5 VIGGREEK I I KHHSLLLLLYYYGLL Cerastotin VIGGAECNINEHRSLVLLYY.... Haly-2 I I GGDECN INEHRS LVLMYY .... Jerdofibrase VI GGDECN INEHPFLVL VYY....

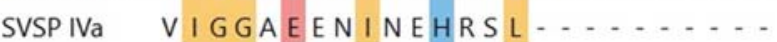

E)

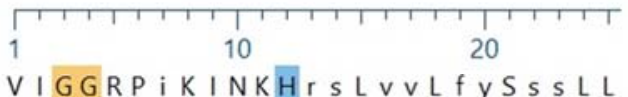

Consensus V I GGRP i K INKHrSLVVLf y S S L L

BmIII-2 VIGGRPIKINKHRRLVVLFKSSSLL

BmIII-3 - IGGRP I K INKHRSLVVLFTSSSLL

BmIll-4 VLGGDPADLNYHPFFAAMMYSP...

BmIII-5 VIGGREEK I I KHHSLLLLLYYYGLL

Figure 3. Multiple alignments of $N$-terminal amino acids sequences of thrombin-like serine proteinases (TLSP) from B. alternatus and B. moojeni venoms, obtained by the Edman degradation method. (A) BmIII-2, (B) BmIII-3, (C) BmIII-4, (D) BmIII-5 and (E) BmIII2-5. The N-terminal sequences of other SVSP were obtained from the BLAST-p database and aligned using ClustalW in Edit Seq 5.01 (C) DNASTAR. (Madison, WI., USA). Colors show only conserved residues compared to reference. PA-BJ from B. jararaca (P81824.2), SP3 from Protobothrops jerdonii (Q9DF66.1), SVSP 5 from T. stejnegeri (Q8AY78.1), GPV-PA from T. albolabris (P0DJF5.1), VLAF from Macrovipera lebetina (Q8JH85.1), Leucurobin from B. leucurus (P0DJ86.1), Bothrombin from B. jararaca (P81661.1), Batroxobin from B. atrox (P04971.1), BjssuSP-1 from B. jararacussu (Q2PQJ3.1), Barnettobin from B. barnetti (K4LLQ2.1), Cerastotin from C. cerastes (P81038.1), Haly-2 from Gloydius brevicaudus (Q9YGJ9.1), Jerdofibrase from Protobothrops jerdonii (P0DMU1.1) and SVSP IVa from C. cerastes (Q7LZF4.1).
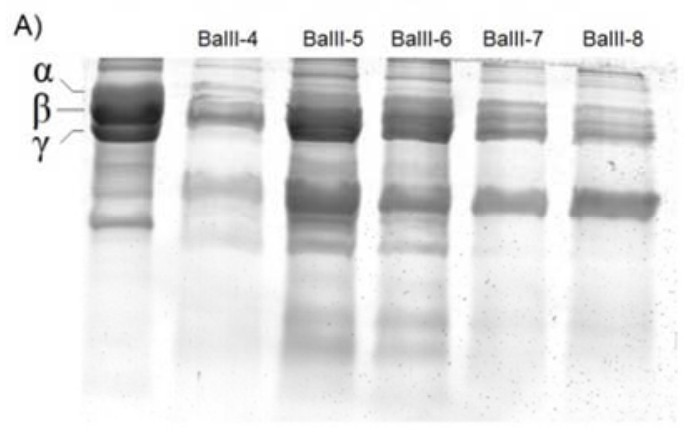

B)

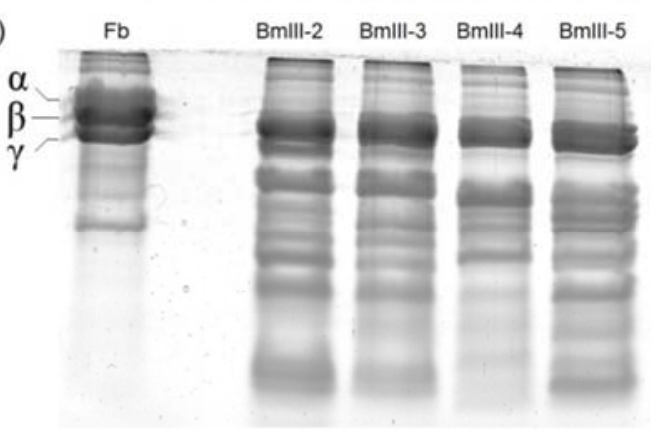

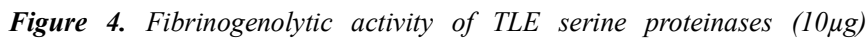
obtained from B. alternatus (A) (BaIII-4, BaIII-5, BaIII-6, BaIII-7, BaIII-8) and B. moojeni (B) (BmIII-2, BmIII-3, BmIII-4, BmIII-5). Bovine fibrinogen (Fb) degradation was analyzed in $13 \%$ SDS-PAGE.

\subsection{Determination Fibrinogenolytic Activity of Thrombin Like Enzymes from B. alternatus and B. moojeni Venoms}

The serine proteinases from B. alternatus (BaIII-4 - 8) as well as those from $B$. moojeni (BmIII-2 - 5) cleaved the bovine fibrinogen $\mathrm{A} \alpha$ (Figure $4 \mathrm{~A}$ and $\mathrm{B}$ respectively). The degradation of the $\mathrm{A} \alpha$ chain was complete.

\section{Discussion}

Purification of snake venoms requires standard handling criteria and techniques. The purified sample must have a high qualitywhich means a certain degree of purity, in addition to maintaining its original biological activity. Therefore, methods of purification should provide the venom sample with the minimum of changes in its composition and, consequently, in its biological activities [5].

The isolation and purification of SVTLEs usually use conventional purification systems, which consist of three chromatographic techniques that combine gel filtration, affinity and ion exchange, such as DEAE-Sephacel, Sephadex G-75 and Benzamidine-Sepharose [11]; Benzamidine-Sepharose, Sephadex G-25 and reversed phase high performance chromatography [46]; Sephacryl S-100, 
Benzamidine-Sepharose and cation-exchange column [17]; Sephacryl S-200, sepharose-agmatine and ion-exchange chromatography on DEAE-Sepharose CL-6B [24]. In this work, SVTLEs from B. moojeni and $B$. alternatus were isolated using an optimized method that is faster than traditional protocols. This new procedure involved two chromatographic steps, combining affinity chromatography (Benzamidine-Seharose 6B) and reverse phase HPLC with high purity degree. Five SVTLEs (designated BaIII-4 - 8) from $B$. alternatus and four SVTLEs (designated BmIII-2 - 5) from $B$. moojeni were purified. All these SVTLEs seem to be homogenous on SDS-PAGE since a single protein band was detected for all these fractions. The relative molecular mass of the purified SVTLEs was 30-40 kDa. Most of SVTLEs isolated from snake venoms were single chain with molecular mass of 26 to $67 \mathrm{kDa}$, depending on their carbohydrate content $[15,22,27]$.

One of the major consequences of using several steps during a protein purification is the loss of material due to freeze-drying intermediate steps. The purification of Leucuribin from B. leucurus, for instance, was described as $36.7 \%$ yield after three chromatographic steps [24]; Agkihpin from $G$. halys Pallas had a yield of $51 \%$ and $5.5 \mathrm{mg}$ of protein were obtained from a gram of venom subjected to four stages of purification [39]; after three chromatographic steps the yield of VLCII from $500 \mathrm{mg}$ of Vipera lebetina venom was about $4.35 \mathrm{mg}$ [3]. The protocol presented in this work allowed us to process half a gram of each venom in a few days with fairly reproducible results. Interestingly, a yield of $51.5 \%$ was reached for BmIII-4 and $16.5 \mathrm{mg}$ of protein was obtained with specific activity increased 30 -fold.

The single bands of SVTLEs were excised from SDSPAGE gels and digested by trypsin digestion followed by ESI-QUAD-TOF Mass Spectrometry and the sequencing of the N-terminal amino acids was performed. The partial sequences of SVTLEs BaIII-5 shared high identity with serine proteinases from other Bothrops snake venoms such as Bhalternin, Asperase, BpirSP27 from B. alternatus, B. asper and $B$. pirajai, respectively $[11,26,29]$. The N-terminal amino acids BaIII-4, BaIII-7 and BaIII-8 showed high identity with SVTLEs of $B$. jararaca (TL-BJ2 and SVSP HS114) [31, 35] and non-bothropic venoms. In addition to Bhalternin other studies have reported thrombin-like enzymes purified from $B$. alternatus venom in three chromatographic steps. Balterobin and SPBA were reported with apparent molecular weight of 30 and $32 \mathrm{kDa}$, respectively, and presence of coagulant and fibrinogenolytic activities [17, 36].

Results obtained in the analysis of triptych ions by mass spectrometry, indicated that the BmIII-4 and BmIII-5 proteins of B. moojeni correspond to SVTLE Batroxobin [21], with a Mascot / Ion relationship Score of 106/47 and 142/53 respectively. Therefore, these results indicate either identity or high structural similarity. Analysis of the alignment of the amino acid residue sequences of the $\mathrm{N}$-terminal of the isolated proteins with other SVTLEs suggest a high identity of BmIII-4 with Leucurobin, Bothrombin and Batroxobin. Proteins BmIII-
2 and 3 with coagulant activity showed high similarity with the enzyme PA-BJ isolated from $B$. jararaca venom which induces platelet aggregation (Serrano, Mentele, Sampaio \& Fink, 1995). BmIII-5 did not show structural similarity with any of the thrombin-like enzymes from botropic venoms deposited in the Mascot and BLASTp / NCBI databases. Other studies have reported the isolation and amino acid sequence of SVTLEs from the B. moojeni venom, BMII32, BMII35 and BmooSP with coagulant / fibrinogenolytic activity and molecular masses of 32,35 and $36 \mathrm{kDa}$, respectively. Additionally, amino acid sequences of those SVTLEs showed high identity with batroxobin (de Oliveira et al., 2016; Fernandes de Oliveira et al., 2013).

According to their specificity for cleaving fibrinogen chains, the serine proteinases have been classified as $\alpha, \beta$ and $\gamma$-fibrinogenases. SVSPs preferentially cleave the $\beta$ b-chain (Menaldo et al., 2012), however, some of them such as Batroxobin from B. atrox, BjussuSP-I from B. jararacussu and Bhalternin from B. alternatus (Costa Jde et al., 2010; Sant' Ana et al., 2008; Stocker \& Barlow, 1976) cleave the A $\alpha$-chain. The latter are considered $\alpha$-fibrinogenases, classical enzymes studied in basic and clinical research. The fibrinogenolitic activity test performed with samples of thrombin-like serine proteinases obtained from $B$. alternatus and $B$. moojen $i$ venoms in this work demonstrated that these enzymes can be considered $\alpha$-fibrinogenases, mainly due to the fact they hydrolyze the A $\alpha$ chain. Curiously, SVTLEs from $B$. moojeni showed greater fibrinogenolitic activity than those from B. alternatus.

Finally, this new purification alternative approach developed using only two steps was faster and more economical than the traditional process. Faster purification and better-improved extraction yield can provide new insights into these enzymes including the use as a candidate molecule in the production of new drugs.

\section{Conclusion}

We have presented an optimized purification protocol to obtain SVTLEs from B. alternatus and B. moojeni venoms only in two steps with a high degree of purity. The enzymes were characterized using SDS-PAGE and in-gel protein digestion coupled to LC-MS/MS and Mascot protein identification, and their coagulant activity was assessed. All SVTLEs had molecular masses of $30-40 \mathrm{kDa}$ and their partial sequences shared high identity with venom plasminogen activator, plateletaggregating enzyme, SVSPs and batroxobin from Bothrops venoms. The SVTLEs purification process developed in this study is faster and more economical compared to other traditional methods. The faster purification and high yield can provide new insights about these enzymes, including the use as a candidate molecule in the production of new drugs.

\section{Authors Contributions}

The authors contributions were Mauricio Aurelio Gomes Heleno developed the conceptualization, methodology, 
formal analysis, writing - original draft preparation, review and funding acquisition. Edda Evnet Newball-Noriega and Salomon Huancahuire-Vega developed the formal analysis, investigation, original draft preparation and writing - editing. Benedito Barraviera and Rui Seabra Ferreira Junior developed the conceptualization, formal analysis, supervision, project administration and review \& editing. All authors approved the submitted version.

\section{Funding}

This work was supported by Coordenação de Aperfeiçoamento de Pessoal de Nível Superior (CAPES; AUXPE Toxinologia 1219/2011, grant no. 23038.0006285/2011-21 and AUXPE grant no. 23038.005536/2012-31) and was part of Postdoctoral research of Mauricio Aurelio Gomes Heleno. RSF Jr. is a CNPq PQ1C fellow researcher [303224/20185].

\section{Conflicts of Interest}

The authors declare that they have no competing interests.

\section{Ethical Statement}

The research protocols used in this study followed the guidelines of the Ethical Committee for use of animals of UNESP, Botucatu, SP, Brazil and international law and policies.

\section{Acknowledgements}

The authors thanks Dr. Cleyton C. Domingues for linguistic support. Special thanks to Dra. Lucilene Delazari dos Santos of the Center for the Study of Venoms and Venomous Animals, CEVAP for collaboration and technical support.

\section{References}

[1] Abbade, L. P. F., Ferreira, R. S., Jr., Dos Santos, L. D., \& Barraviera, B. (2020). Chronic venous ulcers: a review on treatment with fibrin sealant and prognostic advances using proteomic strategies. J Venom Anim Toxins Incl Trop Dis, 26, e20190101. doi: 10.1590/1678-9199-jvatitd-2019-0101.

[2] Altschul, S. F., Madden, T. L., Schäffer, A. A., Zhang, J., Zhang, Z., Miller, W., \& Lipman, D. J. (1997). Gapped BLAST and PSI-BLAST: a new generation of protein database search programs. Nucleic Acids Res, 25 (17), 33893402. doi: $10.1093 /$ nar/25.17.3389.

[3] Amel, K. S., \& Fatima, L. D. (2015). Purification and Characterization of a New Serine Protease (VLCII) Isolated from Vipera lebetina Venom: Its Role in Hemostasis. $J$ Biochem Mol Toxicol, 29 (8), 388-397. doi: 10.1002/jbt.21709.

[4] Amorim, F. G., Menaldo, D. L., Carone, S. E. I., Silva, T. A., Sartim, M. A., De Pauw, E.,... Sampaio, S. V. (2018). New Insights on Moojase, a Thrombin-Like Serine Protease from Bothrops moojeni Snake Venom. Toxins (Basel), 10 (12). doi: $10.3390 /$ toxins 10120500 .
[5] Angulo, Y., \& Lomonte, B. (2009). Biochemistry and toxicology of toxins purified from the venom of the snake Bothrops asper. Toxicon, 54 (7), 949-957. doi: 10.1016/j.toxicon.2008.12.014.

[6] Barros, L. C., Ferreira, R. S., Jr., Barraviera, S. R., Stolf, H. O., Thomazini-Santos, I. A., Mendes-Giannini, M. J., Barraviera, B. (2009). A new fibrin sealant from Crotalus durissus terrificus venom: applications in medicine. J Toxicol Environ Health B Crit Rev, 12 (8), 553-571. doi: 10.1080/10937400903442514.

[7] Buchaim, D. V., Cassaro, C. V., Shindo, J., Coletta, B. B. D., Pomini, K. T., Rosso, M. P. O., Buchaim, R. L. (2019). Unique heterologous fibrin biopolymer with hemostatic, adhesive, sealant, scaffold and drug delivery properties: a systematic review. J Venom Anim Toxins Incl Trop Dis, 25, e20190038. doi: 10.1590/1678-9199-jvatitd-2019-0038.

[8] Cassaro, C. V., Justulin, L. A., Jr., de Lima, P. R., Golim, M. A., Biscola, N. P., de Castro, M. V.,... Barraviera, B. (2019). Fibrin biopolymer as scaffold candidate to treat bone defects in rats. J Venom Anim Toxins Incl Trop Dis, 25, e20190027. doi: 10.1590/1678-9199-jvatitd-2019-0027.

[9] Choi, S. K., Kim, C. W., Kim, J. T., Seomun, Y., Park, M. S., \& Kim, C. O. (2018). Coagulant Effect and Tolerability of Yeast-Produced Recombinant Batroxobin in Healthy Adult Subjects. Clin Drug Investig, 38 (9), 829-835. doi: 10.1007/s40261-018-0673-x.

[10] Costa, F. L., Rodrigues, R. S., Izidoro, L. F., Menaldo, D. L., Hamaguchi, A., Homsi-Brandeburgo, M. I., Rodrigues, V. M. (2009). Biochemical and functional properties of a thrombin-like enzyme isolated from Bothrops pauloensis snake venom. Toxicon, 54 (6), 725-735. doi: 10.1016/j.toxicon.2009.05.040.

[11] Costa, J. O., Fonseca, K. C., Mamede, C. C., Beletti, M. E., Santos-Filho, N. A., Soares, A. M., de Oliveira, F. (2010). Bhalternin: Functional and structural characterization of a new thrombin-like enzyme from Bothrops alternatus snake venom. $\begin{array}{llll}\text { Toxicon, } & 55 & \text { (7), } & \text { 1365-1377. }\end{array}$ 10.1016/j.toxicon.2010.02.014.

[12] Creste, C. F. Z., Orsi, P. R., Landim-Alvarenga, F. C., Justulin, L. A., Golim, M. A., Barraviera, B., \& Ferreira, R. S., Jr. (2020). Highly effective fibrin biopolymer scaffold for stem cells upgrading bone regeneration. Materials (Basel), 13 (12). doi: $10.3390 / \mathrm{ma13122747.}$

[13] de Barros, C. N., Miluzzi Yamada, A. L., Junior, R. S., Barraviera, B., Hussni, C. A., de Souza, J. B.,... Garcia Alves, A. L. (2016). A new heterologous fibrin sealant as a scaffold to cartilage repair-Experimental study and preliminary results. Exp Biol Med (Maywood), 241 (13), 1410-1415. doi: $10.1177 / 1535370215597192$.

[14] de Oliveira, C. T. B., Leonel, B. C., de Oliveira, A. C., de Brito Paiva, M., Ramos, J., Barraviera, B., Shimano, A. C. (2020). Effects of fibrin sealant and bone fragments on defect regeneration performed on rat tibiae: An experimental study. $J$ Mech Behav Biomed Mater, 104, 103662. doi: 10.1016/j.jmbbm.2020.103662.

[15] de Oliveira, F., de Sousa, B. B., Mamede, C. C., de Morais, N. C., de Queiroz, M. R., da Cunha Pereira, D. F.,... Homi Brandeburgo, M. I. (2016). Biochemical and functional characterization of BmooSP, a new serine protease from Bothrops moojeni snake venom. Toxicon, 111, 130-138. doi: 10.1016/j.toxicon.2016.01.055. 
[16] Edman, P., \& Begg, G. (1967). A protein sequenator. Eur $J$ Biochem, 1 (1), 80-91. doi: 10.1007/978-3-662-25813-2_14.

[17] Fernandes de Oliveira, L. M., Ullah, A., Masood, R., Zelanis, A., Spencer, P. J., Serrano, S. M., \& Arni, R. K. (2013). Rapid purification of serine proteinases from Bothrops alternatus and Bothrops moojeni venoms. Toxicon, 76, 282-290. doi: 10.1016/j.toxicon.2013.10.016.

[18] Ferreira, R. S., Jr., de Barros, L. C., Abbade, L. P. F., Barraviera, S., Silvares, M. R. C., de Pontes, L. G., Barraviera, B. (2017). Heterologous fibrin sealant derived from snake venom: from bench to bedside - an overview. $J$ Venom Anim Toxins Incl Trop Dis, 23, 21. doi: 10.1186/s40409-017-0109-8.

[19] Guedes, H. L., Silva, F. P., Jr., Netto, C. C., de Salles, C. M., Alexandre, G., Oliveira, C. L., De Simone, S. G. (2008). Structural characterization and low-resolution model of BJ-48, a thrombin-like enzyme from Bothrops jararacussu venom. Biophys Chem, 132 (2-3), 159-164. doi: 10.1016/j.bpc.2007.11.002.

[20] Iatecola, A., Barraviera, B., Ferreira, R. S., Jr., dos Santos, G. R., Neves, J. I., \& da Cunha, M. R. (2013). Use of a new fibrin sealant and laser irradiation in the repair of skull defects in rats. Braz Dent J, 24 (5), 456-461. doi: 10.1590/01036440201302265 .

[21] Itoh, N., Tanaka, N., Mihashi, S., Yamashina, I. (1987). Molecular cloning and sequence analysis of cDNA for batroxobin, a thrombin-like snake venom enzyme. J Biol Chem. 5; 262 (7): 3132-5. PMID: 3546302.

[22] Kadi-Saci, A., \& Laraba-Djebari, F. (2020). Purification and characterization of a thrombin-like enzyme isolated from Vipera lebetina venom: its interaction with platelet receptor. Blood Coagul Fibrinolysis, 31 (1), 1-10. doi: $10.1097 / \mathrm{mbc} .0000000000000856$.

[23] Kini, R. M. (2005). Serine proteases affecting blood coagulation and fibrinolysis from snake venoms. Pathophysiol Haemost Thromb, 34 (4-5), 200-204. doi: 10.1159/000092424.

[24] Magalhães, A., Magalhães, H. P., Richardson, M., Gontijo, S., Ferreira, R. N., Almeida, A. P., \& Sanchez, E. F. (2007). Purification and properties of a coagulant thrombin-like enzyme from the venom of Bothrops leucurus. Comp Biochem Physiol A Mol Integr Physiol, 146 (4), 565-575. doi: 10.1016/j.cbpa.2005.12.033.

[25] Marsh, N., \& Williams, V. (2005). Practical applications of snake venom toxins in haemostasis. Toxicon, 45 (8), 11711181. doi: 10.1016/j.toxicon.2005.02.016.

[26] Menaldo, D. L., Bernardes, C. P., Santos-Filho, N. A., Moura Lde, A., Fuly, A. L., Arantes, E. C., \& Sampaio, S. V. (2012). Biochemical characterization and comparative analysis of two distinct serine proteases from Bothrops pirajai snake venom. Biochimie, $94 \quad$ (12), 2545-2558. doi: 10.1016/j.biochi.2012.07.007.

[27] Mukherjee, A. K. (2014). The pro-coagulant fibrinogenolytic serine protease isoenzymes purified from Daboia russelii russelii venom coagulate the blood through factor $\mathrm{V}$ activation: role of glycosylation on enzymatic activity. PLoS One, 9 (2), e86823. doi: 10.1371/journal.pone.0086823.

[28] Nielsen, V. G., Boyer, L. V., Redford, D. T., \& Ford, P. (2017). Thrombelastographic characterization of the thrombin-like activity of Crotalus simus and Bothrops asper venoms. Blood Coagul Fibrinolysis, 28 (3), 211-217. doi: $10.1097 / \mathrm{mbc} .0000000000000577$.

[29] Pérez, A. V., Rucavado, A., Sanz, L., Calvete, J. J., \& Gutiérrez, J. M. (2008). Isolation and characterization of a serine proteinase with thrombin-like activity from the venom of the snake Bothrops asper. Braz J Med Biol Res, 41 (1), 1217. doi: $10.1590 / \mathrm{s} 0100-879 \times 2006005000189$.

[30] Rodrigues, V. M., Soares, A. M., Guerra-Sá, R., Rodrigues, V., Fontes, M. R., \& Giglio, J. R. (2000). Structural and functional characterization of neuwiedase, a nonhemorrhagic fibrin(ogen)olytic metalloprotease from Bothrops neuwiedi snake venom. Arch Biochem Biophys, 381 (2), 213-224. doi: 10.1006/abbi.2000.1958.

[31] Saguchi, K., Hagiwara-Saguchi, Y., Murayama, N., Ohi, H., Fujita, Y., Camargo, A. C., Higuchi, S. (2005). Molecular cloning of serine proteinases from Bothrops jararaca venom gland. Toxicon, 46 (1), 72-83. doi: 10.1016/j.toxicon.2005.03.011.

[32] Sant' Ana, C. D., Ticli, F. K., Oliveira, L. L., Giglio, J. R., Rechia, C. G., Fuly, A. L.,... Sampaio, S. V. (2008). BjussuSP-I: a new thrombin-like enzyme isolated from Bothrops jararacussu snake venom. Comp Biochem Physiol A Mol Integr Physiol, 151 (3), 443-454. doi: 10.1016/j.cbpa.2007.02.036

[33] Sartori Filho, R., Prestes, N. C., Thomazini, I. A., MendesGiannini, M. J., Toscano, E., Canavessi, A. M. O., \& Barraviera, B. (1998). Use of fibrin glue derived from snake venom in testicular biopsy of rams. Journal of Venomous Animals and Toxins, 4, 23-35. Retrieved from http://www.scielo.br/scielo.php?script=sci_arttext\&pid=S010 4-79301998000100003\&nrm=iso.

[34] Serrano, S. M. (2013). The long road of research on snake venom serine proteinases. Toxicon, 62, 19-26. doi: 10.1016/j.toxicon.2012.09.003.

[35] Serrano, S. M., Sampaio, C. A., Mentele, R., Camargo, A. C., \& Fink, E. (2000). A novel fibrinogen-clotting enzyme, TL-BJ, from the venom of the snake Bothrops jararaca: purification and characterization. Thromb Haemost, 83 (3), 438-444.

[36] Smolka, M. B., Marangoni, S., Oliveira, B., \& Novello, J. C. (1998). Purification and partial characterization of a thrombinlike enzyme, balterobin, from the venom of Bothrops alternatus. Toxicon, 36 (7), 1059-1063. doi: 10.1016/s00410101(98)80008-1.

[37] Stocker, K., \& Barlow, G. H. (1976). The coagulant enzyme from Bothrops atrox venom (batroxobin). Methods Enzymol, 45, 214-223. doi: 10.1016/s0076-6879(76)45021-8.

[38] Swenson, S., \& Markland, F. S., Jr. (2005). Snake venom fibrin(ogen)olytic enzymes. Toxicon, 45 (8), 1021-1039. doi: 10.1016/j.toxicon.2005.02.027.

[39] Tang, Y., Huang, M., Hu, Q., Wu, H., Yao, J., Sun, K., \& Li, X. (2018). Agkihpin, a Distinct SVTLE from the Venom of Gloydius halys Pallas: Purification, Characterization and Structure-Activity Determination. Chem Biodivers, 15 (6), e1800122. doi: 10.1002/cbdv.201800122.

[40] Theakston, R. D., \& Reid, H. A. (1983). Development of simple standard assay procedures for the characterization of snake venom. Bull World Health Organ, 61 (6), 949-956. 
[41] Ullah, A., Masood, R., Ali, I., Ullah, K., Ali, H., Akbar, H., \& Betzel, C. (2018). Thrombin-like enzymes from snake venom: Structural characterization and mechanism of action. Int J Biol Macromol, 114, 788-811. doi: 10.1016/j.ijbiomac.2018.03.164.

[42] Vieira, D. F., Watanabe, L., Sant'ana, C. D., Marcussi, S., Sampaio, S. V., Soares, A. M., \& Arni, R. K. (2004). Purification and characterization of jararassin-I, A thrombinlike enzyme from Bothrops jararaca snake venom. Acta Biochim Biophys Sin (Shanghai), 36 (12), 798-802. doi: 10.1093/abbs/36.12.798

[43] Vivas-Ruiz, D. E., Sandoval, G. A., Gonzalez-Kozlova, E., Zarria-Romero, J., Lazo, F., Rodríguez, E., Sanchez, E. F. (2020). Fibrinogen-clotting enzyme, pictobin, from Bothrops pictus snake venom. Structural and functional characterization. Int J Biol Macromol, 153, 779-795. doi: 10.1016/j.ijbiomac.2020.03.055.
[44] Vivas-Ruiz, D. E., Sandoval, G. A., Mendoza, J., Inga, R. R., Gontijo, S., Richardson, M.,... Sanchez, E. F. (2013). Coagulant thrombin-like enzyme (barnettobin) from Bothrops barnetti venom: molecular sequence analysis of its cDNA and biochemical properties. Biochimie, 95 (7), 1476-1486. doi: 10.1016/j.biochi.2013.03.015.

[45] You, W. K., Choi, W. S., Koh, Y. S., Shin, H. C., Jang, Y., \& Chung, K. H. (2004). Functional characterization of recombinant batroxobin, a snake venom thrombin-like enzyme, expressed from Pichia pastoris. FEBS Lett, 571 (1-3), 67-73. doi: 10.1016/j.febslet.2004.06.060.

[46] Zaqueo, K. D., Kayano, A. M., Domingos, T. F., Moura, L. A., Fuly, A. L., da Silva, S. L.,... Soares, A. M. (2016). BbrzSP-32, the first serine protease isolated from Bothrops brazili venom: Purification and characterization. Comp Biochem Physiol A Mol Integr Physiol, 195, 15-25. doi: 10.1016/j.cbpa.2016.01.021. 